\title{
Nontrivial Solutions for Time Fractional Nonlinear Schrödinger-Kirchhoff Type Equations
}

\author{
N. Nyamoradi, ${ }^{1}$ Y. Zhou, ${ }^{2,3}$ E. Tayyebi, ${ }^{1}$ B. Ahmad, ${ }^{3}$ and A. Alsaedi ${ }^{3}$ \\ ${ }^{1}$ Department of Mathematics, Faculty of Sciences, Razi University, Kermanshah 67149, Iran \\ ${ }^{2}$ Faculty of Mathematics and Computational Science, Xiangtan University, Hunan 411105, China \\ ${ }^{3}$ Nonlinear Analysis and Applied Mathematics (NAAM) Research Group, Faculty of Science, King Abdulaziz University, \\ Jeddah 21589, Saudi Arabia
}

Correspondence should be addressed to Y. Zhou; yzhou@xtu.edu.cn

Received 4 April 2017; Revised 11 June 2017; Accepted 22 June 2017; Published 27 July 2017

Academic Editor: Thabet Abdeljawad

Copyright (C) 2017 N. Nyamoradi et al. This is an open access article distributed under the Creative Commons Attribution License, which permits unrestricted use, distribution, and reproduction in any medium, provided the original work is properly cited.

We study the existence of solutions for time fractional Schrödinger-Kirchhoff type equation involving left and right Liouville-Weyl fractional derivatives via variational methods.

\section{Introduction}

In recent years, there has been a great interest in studying problems involving fractional Schrödinger equations [15], Kirchhoff type equations [6-8], fractional Navier-Stokes equations $[9,10]$, and fractional ordinary differential equations and Hamiltonian systems [11-17], and so forth. For further details and applications, we refer the reader to $[18,19]$ and the references cited therein.

On the other hand, the integer-order SchrödingerKirchhoff type equations have also been investigated by many authors; for example, see [20-23]. In fact, SchrödingerKirchhoff type equations play an important role in modelling several physical and biological systems. However, to the best of our knowledge, the existence of solutions to the time fractional Schrödinger-Kirchhoff type equations has yet to be addressed.

The objective of the present paper is to study time fractional Schrödinger-Kirchhoff type equation of the form

$$
\begin{gathered}
\left(a+\left.\left.b \int_{\mathbb{R}}\right|_{-\infty} D_{t}^{\alpha} u(t)\right|^{2} d t\right)^{\theta-1}{ }_{t} D_{\infty}^{\alpha}\left({ }_{-\infty} D_{t}^{\alpha} u(t)\right) \\
+\mu V(t) u=f(t, u), \quad t \in \mathbb{R}, u \in H^{\alpha}(\mathbb{R}),
\end{gathered}
$$

where $\alpha \in(1 / 2,1],{ }_{-\infty} D_{t}^{\alpha}$ and ${ }_{t} D_{\infty}^{\alpha}$, respectively, denote left and right Liouville-Weyl fractional derivatives of order $\alpha$ on
$\mathbb{R}, a, b>0$ are constants, $\mu>0$ is parameter, $\theta>1, f \in$ $C(\mathbb{R} \times \mathbb{R}, \mathbb{R})$, and $V: \mathbb{R} \rightarrow \mathbb{R}^{+}$is a potential function.

The rest of the paper is organized as follows. Section 2 contains preliminary concepts of fractional calculus and fractional Sobolev space, while some important lemmas, which are needed in the proof of main results, are obtained in Section 3. We present our main results in Section 4.

\section{Preliminaries}

In this section, we recall important definitions and concepts of fractional calculus and then prove certain results about fractional Sobolev space $H^{\alpha}(\mathbb{R})$ related to our study of the problem at hand.

Definition 1 (see [24]). The left and right Liouville-Weyl fractional integrals of order $\alpha \in(0,1)$ on $\mathbb{R}$ are defined by

$$
\begin{aligned}
{ }_{-\infty} I_{x}^{\alpha} \phi(x) & =\frac{1}{\Gamma(\alpha)} \int_{-\infty}^{x}(x-\xi)^{\alpha-1} \phi(\xi) d \xi, \\
{ }_{x} I_{\infty}^{\alpha} \phi(x) & =\frac{1}{\Gamma(\alpha)} \int_{x}^{\infty}(\xi-x)^{\alpha-1} \phi(\xi) d \xi,
\end{aligned}
$$

respectively, where $x \in \mathbb{R}$. 
The left and right Liouville-Weyl fractional derivatives of order $\alpha \in(0,1)$ on $\mathbb{R}$ are defined by

$$
\begin{aligned}
{ }_{-\infty} D_{x}^{\alpha} \phi(x) & =\frac{d}{d x}-\infty I_{x}^{1-\alpha} \phi(x), \\
{ }_{x} D_{\infty}^{\alpha} \phi(x) & =-\frac{d}{d x} x_{\infty}^{1-\alpha} \phi(x),
\end{aligned}
$$

respectively, where $x \in \mathbb{R}$.

The definitions (3) may be written in an alternative form as follows:

$$
\begin{aligned}
{ }_{-\infty} D_{x}^{\alpha} \phi(x) & =\frac{\alpha}{\Gamma(1-\alpha)} \int_{0}^{\infty} \frac{\phi(x)-\phi(x-\xi)}{\xi^{\alpha+1}} d \xi \\
{ }_{x} D_{\infty}^{\alpha} \phi(x) & =\frac{\alpha}{\Gamma(1-\alpha)} \int_{0}^{\infty} \frac{\phi(x)-\phi(x+\xi)}{\xi^{\alpha+1}} d \xi .
\end{aligned}
$$

Also, we define the Fourier transform $\mathscr{F}(u)(\xi)$ of $u(x)$ as

$$
\mathscr{F}(u)(\xi)=\int_{-\infty}^{\infty} e^{-i x \cdot \xi} u(x) d x .
$$

For any $\alpha>0$, we define the seminorm and norm, respectively, as [16]

$$
\begin{aligned}
|u|_{I_{-\infty}^{\alpha}} & =\left\|{ }_{-\infty} D_{x}^{\alpha} u\right\|_{L^{2}}, \\
\|u\|_{I_{-\infty}^{\alpha}} & =\left(\|u\|_{L^{2}}^{2}+|u|_{I_{-\infty}^{\alpha}}^{2}\right)^{1 / 2},
\end{aligned}
$$

and let the space $I_{-\infty}^{\alpha}(\mathbb{R})$ denote the completion of $C_{0}^{\infty}(\mathbb{R})$ with respect to the norm $\|\cdot\|_{I_{-\infty}^{\alpha}}$.

Next, for $0<\alpha<1$, we give the relationship between classical fractional Sobolev space $H^{\alpha}(\mathbb{R})$ and $I_{-\infty}^{\alpha}(\mathbb{R})$, where $H^{\alpha}(\mathbb{R})$ is defined by

$$
H^{\alpha}(\mathbb{R})={\overline{C_{0}^{\infty}(\mathbb{R})}}^{\|\cdot\|_{\alpha}},
$$

with the norm

$$
\|u\|_{\alpha}=\left(\|u\|_{L^{2}}^{2}+|u|_{\alpha}^{2}\right)^{1 / 2}
$$

and seminorm

$$
|u|_{\alpha}=\left\||\xi|^{\alpha} \mathscr{F}(u)\right\|_{L^{2}} .
$$

Observe that the spaces $H^{\alpha}(\mathbb{R})$ and $I_{-\infty}^{\alpha}(\mathbb{R})$ are equal and have equivalent norms (see [16]).

Therefore, we define

$$
H^{\alpha}(\mathbb{R})=\left\{\left.u \in L^{2}(\mathbb{R})|| \xi\right|^{\alpha} \mathscr{F}(u) \in L^{2}(\mathbb{R})\right\} .
$$

Let

$$
\begin{aligned}
X^{\alpha} & =\left\{u \in H^{\alpha}(\mathbb{R}) \mid \int_{\mathbb{R}}\left(\left|{ }_{-\infty} D_{t}^{\alpha} u(t)\right|^{2}+|u(t)|^{2}\right) d t\right. \\
& <\infty\} .
\end{aligned}
$$

The space $X^{\alpha}$ is a reflexive and separable Hilbert space with the inner product

$$
\begin{aligned}
& \langle u, v\rangle_{X^{\alpha}} \\
& \quad=\int_{\mathbb{R}}\left({ }_{-\infty} D_{t}^{\alpha} u(t) \cdot{ }_{-\infty} D_{t}^{\alpha} v(t)+u(t) v(t)\right) d t
\end{aligned}
$$

and the corresponding norm

$$
\|u\|_{X^{\alpha}}^{2}=\langle u, u\rangle_{X^{\alpha}} .
$$

Define the space

$$
X_{\mu}^{\alpha}=\left\{u \in X^{\alpha}: \int_{\mathbb{R}} \mu V(t)|u|^{2} d t<+\infty\right\},
$$

with the norm

$$
\begin{gathered}
\|u\|_{X_{\mu}^{\alpha}}=\left(\int_{\mathbb{R}} a^{\theta-1}\left(\left|{ }_{-\infty} D_{t}^{\alpha} u(t)\right|^{2}\right) d t\right. \\
\left.+\int_{\mathbb{R}} \mu V(t)|u|^{2} d t\right)^{1 / 2} .
\end{gathered}
$$

Lemma 2. $\left(X_{\mu}^{\alpha},\|\cdot\|_{X_{\mu}^{\alpha}}\right)$ is a uniformly convex Banach space.

Proof. $X_{\mu}^{\alpha}$ is obviously Banach space. Now, we can prove that $\left(X_{\mu}^{\alpha},\|\cdot\|_{X_{\mu}^{\alpha}}\right)$ is uniformly convex. To this end, let $0<\varepsilon<2$ and $u, v \in X_{\mu}^{\alpha}$ with $\|u\|_{X_{\lambda}^{\alpha}}=\|v\|_{X_{\mu}^{\alpha}}=1$ and $\|u-v\|_{X_{\mu}^{\alpha}} \geq \varepsilon$. Using the following inequality:

$$
\left|\frac{a+b}{2}\right|^{2}+\left|\frac{a-b}{2}\right|^{2} \leq \frac{1}{2}\left(|a|^{2}+|b|^{2}\right), \quad \forall a, b \in \mathbb{R},
$$

we get

$$
\begin{aligned}
& \left\|\frac{u+v}{2}\right\|_{X_{\mu}^{\alpha}}^{2}+\left\|\frac{u-v}{2}\right\|_{X_{\mu}^{\alpha}}^{2} \\
& \quad=\int_{\mathbb{R}} a^{\theta-1}\left(\left|{ }_{-\infty} D_{t}^{\alpha}\left(\frac{u+v}{2}\right)(t)\right|^{2}\right) d t \\
& +\int_{\mathbb{R}} \mu V(t)\left|\frac{u+v}{2}\right|^{2} d t \\
& +\int_{\mathbb{R}} a^{\theta-1}\left(\left|-\infty D_{t}^{\alpha}\left(\frac{u-v}{2}\right)(t)\right|^{2}\right) d t \\
& +\int_{\mathbb{R}} \mu V(t)\left|\frac{u-v}{2}\right|^{2} d t \\
& \quad \leq \frac{1}{2}\left(\int_{\mathbb{R}} a^{\theta-1}\left(\left|-\infty D_{t}^{\alpha} u(t)\right|^{2}\right) d t\right. \\
& +\int_{\mathbb{R}} a^{\theta-1}\left(\left|-\infty D_{t}^{\alpha} v(t)\right|^{2}\right) d t+\int_{\mathbb{R}} \mu V(t)|u|^{2} d t \\
& \left.\quad+\int_{\mathbb{R}} \mu V(t)|v|^{2} d t\right)=\frac{1}{2}\left(\|u\|_{X_{\mu}^{\alpha}}^{2}+\|v\|_{X_{\mu}^{\alpha}}^{2}\right)=1,
\end{aligned}
$$

which implies that $\|(u+v) / 2\|_{X_{\mu}^{\alpha}}^{2} \leq 1-\varepsilon / 2$. Hence, taking $\delta=\delta(\varepsilon)$ such that $1-\varepsilon / 2=1-\delta$, we have $\|(u+v) / 2\|_{X_{\mu}^{\alpha}}^{2} \leq 1-\delta$. Therefore, $\left(X_{\mu}^{\alpha},\|\cdot\|_{X_{\mu}^{\alpha}}\right)$ is uniformly convex. 
In the sequel, we need the following assumptions.

(V1) $V(t) \in C(\mathbb{R}, \mathbb{R}), V_{0}:=\inf _{t \in \mathbb{R}} V(t)>0$;

(V2) there exists $r>0$ such that, for any $M>0$,

$$
\text { meas }(\{t \in(y-r, y+r): V(t) \leq M\}) \longrightarrow 0
$$$$
\text { as }|y| \longrightarrow \infty \text {; }
$$

(V3) there exists $l_{0}>0$ such that $\int_{|t| \geq l_{0}} V(t)^{-1} d t<\infty$;

(F1) $f \in C(\mathbb{R} \times \mathbb{R}, \mathbb{R})$ and there exist constants $c_{0}, c_{1}$, $\ldots, c_{l}>0$ and $q_{j} \in(2,2 \theta)$ such that

$$
|f(t, u)| \leq c_{0}|u|+\sum_{j=1}^{l} c_{j}|u|^{q_{j}-1}, \quad \forall(t, u) \in \mathbb{R} \times \mathbb{R} ;
$$

(F2) $f(t, u)=o(|u|)$ as $|u| \rightarrow 0$ uniformly in $t \in \mathbb{R}^{N}$;

(F3) there exist $\lambda \in(2 \theta, \infty)$ such that

$$
\lambda F(t, u) \leq f(t, u) u, \quad \forall t \in \mathbb{R}, u \in \mathbb{R}
$$

(F4) $F(t, u) /|u|^{2 \theta} \rightarrow+\infty$ as $|u| \rightarrow+\infty$ uniformly in $t \in \mathbb{R}$;

(F5) $f(t,-u)=-f(t, u)$ for all $(t, u) \in \mathbb{R} \times \mathbb{R}$;

(F6) $f \in C(\mathbb{R} \times \mathbb{R}, \mathbb{R})$ and there exists $1<p<2$ such that

$$
|f(t, u)| \leq|u|^{p-1}, \quad \forall(t, u) \in \mathbb{R} \times \mathbb{R} ;
$$

(F7) there exist $\sigma_{1}>0,0<\sigma_{2}<1 / 8 D_{2}^{2}\left(D_{2}\right.$ is defined in Remark 6), $1 \leq \gamma<2$, and small constants $0<r_{0}<r_{1}$ such that

$$
\begin{aligned}
& \sigma_{1}|u|^{\gamma}<F(t, u) \leq \sigma_{2}|u|^{2}, \\
& \qquad r_{0} \leq|u| \leq r_{1} \text {, a.e. } t \in \mathbb{R} .
\end{aligned}
$$

Lemma 3. Assume that (V1) holds. Then the embeddings $X_{\mu}^{\alpha} \hookrightarrow X^{\alpha} \hookrightarrow L^{2}(\mathbb{R})$ are continuous. In particular, there exists a constant $C_{2}>0$ such that

$$
\|u\|_{L^{2}(\mathbb{R})} \leq C_{2}\|u\|_{X_{\mu}^{\alpha}} \quad \forall u \in X_{\mu}^{\alpha}
$$

Moreover, if (V1) and (V2) hold, then the embedding $X_{\mu}^{\alpha} \hookrightarrow$ $L^{2}(\mathbb{R})$ is compact.

Proof. Clearly, the chain of embeddings $X_{\mu}^{\alpha} \hookrightarrow X^{\alpha} \hookrightarrow L^{2}(\mathbb{R})$ is continuous and consequently one can obtain (23). Also in view of (V1), (V2), and following the method of proof similar to that of Lemma 2.2 in [15], the embedding $X_{\mu}^{\alpha} \hookrightarrow L^{2}(\mathbb{R})$ is compact.

Lemma 4. Let $\alpha>1 / 2$. Then $H^{\alpha}(\mathbb{R}) \subset C(\mathbb{R})$ and there exists a constant $C=C_{\alpha}$ such that

$$
\sup _{x \in \mathbb{R}}|u(x)| \leq C\|u\|_{X_{\mu}^{\alpha}} .
$$

Proof. The proof is similar to that of Theorem 2.1 in [16], so we omit it.

Also by Lemma 4 , there is a constant $C_{\alpha}>0$ such that

$$
\|u\|_{\infty} \leq C_{\alpha}\|u\|_{X_{\mu}^{\alpha}}
$$

Remark 5. If $u \in H^{\alpha}(\mathbb{R})$ with $1 / 2<\alpha<1$, then it follows by Lemma 4 that $u \in L^{q}(\mathbb{R})$ for all $q \in[2, \infty)$ as

$$
\int_{\mathbb{R}}|u(x)|^{q} d x \leq\|u\|_{\infty}^{q-2}\|u\|_{L^{2}(\mathbb{R})}^{2} .
$$

Remark 6. From Remark 5 and Lemma 3, it is easy to verify that the imbedding of $X_{\mu}^{\alpha}$ in $L^{q}(\mathbb{R})$ is also compact for $q \epsilon$ $(2, \infty)$. Hence, for all $2 \leq q<\infty$, the imbedding of $X_{\mu}^{\alpha}$ in $L^{q}(\mathbb{R})$ is continuous and compact, which together with Lemma 4 implies that there exists $D_{q}>0$ such that

$$
\|u\|_{L^{q}(\mathbb{R})} \leq D_{q}\|u\|_{X_{\mu}^{\alpha}} .
$$

Lemma 7. Assume that (V1) and (V3) hold. Then the embed$\operatorname{ding} X_{\mu}^{\alpha} \hookrightarrow L^{p}(\mathbb{R})$ is continuous and compact for $p \in[1,+\infty)$.

Proof. By (V3) and Hölder's inequality, we have

$$
\begin{aligned}
& \int_{|t| \geq l_{0}}|u(t)| d t \\
& \leq\left(\int_{|t| \geq l_{0}} V(t)|u(t)|^{2} d t\right)^{1 / 2}\left(\int_{|t| \geq l_{0}} V(t)^{-1 / 2} d t\right)^{1 / 2} \\
& \leq c_{1}\|u\|_{X_{\mu}^{\alpha}}
\end{aligned}
$$

for some positive constant $c_{1}$. So Lemma 4 implies that

$$
\begin{aligned}
\|u\|_{1} & =\int_{-l_{0}}^{l_{0}}|u(t)| d t+\int_{|t| \geq l_{0}}|u(t)| d t \\
& \leq 2 l_{0}\|u\|_{\infty}+c_{1}\|u\|_{X_{\mu}^{\alpha}} \leq c_{2}\|u\|_{X_{\mu}^{\alpha}},
\end{aligned}
$$

for some positive constant $c_{2}$. Hence, by Remark 6 , we can get continuous embeddings $X_{\mu}^{\alpha}$ into $L^{p}(\mathbb{R})$ for $p \in[1,+\infty)$. Now, we will show that the embedding is compact for $p \in[1,+\infty)$. Let $\left\{u_{n}\right\} \subset X_{\mu}^{\alpha}$ such that $u_{n} \rightarrow 0$ and $M>0$ such that $\|u\|_{X_{\mu}^{\alpha}} \leq$ $M$. In view of (V3), given $\varepsilon>0$, for $l>0$ large enough, one can obtain

$$
\int_{|t| \geq l_{0}} V(t)^{-1 / 2} d t<\left(\frac{\varepsilon}{2 M}\right)^{2}
$$

Then,

$$
\begin{aligned}
& \int_{|t| \geq l}|u(t)| d t \\
& \leq\left(\int_{|t| \geq l} V(t)|u(t)|^{2} d t\right)^{1 / 2}\left(\int_{|t| \geq l} V(t)^{-1 / 2} d t\right)^{1 / 2} \\
& \leq \frac{\varepsilon}{2 M}\|u\|_{X_{\mu}^{\alpha}} \leq \frac{\varepsilon}{2} .
\end{aligned}
$$


On the other hand, by Sobolev's theorem (see, e.g., [25]) which implies that $u_{n} \rightarrow 0$ uniformly on $[-l, l]$, there is $n_{0}$ such that $\int_{-l}^{l}|u(t)| d t<\varepsilon / 2$ for all $n \geq n_{0}$. Thus $u_{n} \rightarrow 0$ in $L^{1}(\mathbb{R})$. So, for $1<p<\infty$, we have

$$
\int_{\mathbb{R}}|u(x)|^{p} d x \leq\|u\|_{\infty}^{p-1} \int_{\mathbb{R}}|u(t)| d t \leq c_{3}\|u\|_{1} \longrightarrow 0
$$

and consequently, $u_{n} \rightarrow 0$ in $L^{p}(\mathbb{R})$ for $p \in[1,+\infty)$.

Definition 8. Let $X$ be a Banach space, $I \in C^{1}(X, \mathbb{R})$. One says that $I$ satisfies the Palais-Smale (PS) condition if any sequence $\left(u_{n}\right) \in X$ for which $I\left(u_{n}\right)$ is bounded and $I^{\prime}\left(u_{n}\right) \rightarrow 0$ as $n \rightarrow$ $\infty$ possesses a convergent subsequence.

In order to establish the main results, we need the following known Theorems.

Theorem 9 (see [26, Theorem 2.2]). Let $X$ be a real Banach space and $I \in C^{1}(X, \mathbb{R})$ satisfies $(P S)$ condition. Suppose $I(0)=$ 0 and

(i) there are constants $\rho, \alpha>0$ such that $\left.I\right|_{\partial B_{\rho}(0)} \geq 0$;

(ii) there is an $e \in X \backslash \overline{B_{\rho}(0)}$ such that $I(e) \leq 0$.

Then I possesses a critical value $c \geq \alpha$. Moreover $c$ can be characterized as

$$
\left.c=\inf _{\gamma \in \Gamma \in[0,1]} \max _{s \in[} I(\gamma)\right),
$$

where

$$
\Gamma=\{\gamma \in C([0,1], X): \gamma(0)=0, \gamma(1)=e\} .
$$

Theorem 10 (see [26, Theorem 9.12]). Let $X$ be an infinite dimensional Banach space and let $I \in C^{1}(X, \mathbb{R})$ be even, satisfying $(P S)$ condition, and $I(0)=0$. If $X=Y \oplus Z$, where $Y$ is finite dimensional and I satisfies the following conditions:

(I1) there exist constants $\rho, \alpha>0$ such that $\left.I\right|_{\partial B_{\rho} \cap Z} \geq \alpha$;

(I2) for any finite dimensional subspace $\widetilde{X} \subset X$, there is $R=R(\widetilde{X})>0$ such that $I(u) \leq 0$ on $\widetilde{X} \backslash B_{R}$,

then I possesses an unbounded sequence of critical values.

\section{Some Lemmas}

Recall that $u \in X_{\mu}^{\alpha}$ is said to be a weak solution of problem (1) if

$$
\begin{array}{r}
\left(a+b \int_{\mathbb{R}}\left|{ }_{-\infty} D_{t}^{\alpha} u(t)\right|^{2} d t\right)^{\theta-1} \\
\cdot \int_{\mathbb{R}}-\infty D_{t}^{\alpha} u(t) \cdot{ }_{-\infty} D_{t}^{\alpha} \varphi(t) d t \\
+\int_{\mathbb{R}} \mu V(t) u(t) \varphi(t) d t=\int_{\mathbb{R}} f(t, u(t)) \varphi(t) d t, \\
\forall \varphi \in X_{\mu}^{\alpha},
\end{array}
$$

and the energy functional $I_{\mu, \theta}: X_{\mu}^{\alpha} \rightarrow \mathbb{R}$ is given by the formula

$$
\begin{aligned}
I_{\mu, \theta}(u)= & \frac{1}{2 b \theta}\left(a+b \int_{\mathbb{R}}\left|{ }_{-\infty} D_{t}^{\alpha} u(t)\right|^{2} d t\right)^{\theta} \\
& +\frac{1}{2} \int_{\mathbb{R}} \mu V(x)|u(t)|^{2} d t \\
& -\int_{\mathbb{R}} F(t, u(t)) d t,
\end{aligned}
$$

where $F(x, u)=\int_{0}^{u} f(t, s) d s$.

In view of assumptions (V1) and (F1), the functional $I_{\mu, \theta}$ is of class $C^{1}\left(X_{\mu}^{\alpha}, \mathbb{R}\right)$ and by similar method in Theorem 4.1 in [27] and the definition of Gâteaux derivative, one can get

$$
\begin{aligned}
\left\langle I_{\mu, \theta}^{\prime}(u), \varphi\right\rangle= & \left(a+b \int_{\mathbb{R}}\left|{ }_{-\infty} D_{t}^{\alpha} u(t)\right|^{2} d t\right)^{\theta-1} \\
& \cdot \int_{\mathbb{R}}-\infty D_{t}^{\alpha} u(t) \cdot{ }_{-\infty} D_{t}^{\alpha} \varphi(t) d t \\
& +\int_{\mathbb{R}} \mu(t) u(t) \varphi(t) d t \\
& -\int_{\mathbb{R}} f(t, u(t)) \varphi(t) d t,
\end{aligned}
$$

$$
\forall u, \varphi \in X_{\mu}^{\alpha}
$$

Lemma 11. Assume that (V) and (F1)-(F3) hold. Then $I_{\mu, \theta}$ satisfies the (PS) condition.

Proof. Let $\left\{u_{n}\right\}_{n \in \mathbb{N}} \subset X_{\mu}^{\alpha}$ be a sequence such that $\left\{I_{\mu, \theta}\left(u_{n}\right)\right\}_{n \in \mathbb{N}}$ is bounded and $I_{\mu, \theta}^{\prime}\left(u_{n}\right) \rightarrow 0$ as $n \rightarrow \infty$. Then there exits $D>0$ such that $\left|\left\langle I_{\mu, \theta}^{\prime}\left(u_{n}\right), u_{n}\right\rangle\right| \leq D\|u\|_{X_{\mu}^{\alpha}}$ and $\left|I_{\mu, \theta}\left(u_{n}\right)\right| \leq D$. So, by (F3), (23), and the fact that $\lambda>2 \theta>1$, we get

$$
\begin{aligned}
\lambda D & +D\|u\|_{X_{\mu}^{\alpha}} \geq \lambda I_{\mu, \theta}\left(u_{n}\right)-\left\langle I_{\mu, \theta}^{\prime}\left(u_{n}\right), u_{n}\right\rangle \\
& =\frac{\lambda}{2 b \theta}\left(a+\left.\left.b \int_{\mathbb{R}}\right|_{-\infty} D_{t}^{\alpha} u_{n}(t)\right|^{2} d t\right)^{\theta}+\frac{\lambda}{2} \\
& \cdot \int_{\mathbb{R}} \mu V(t)\left|u_{n}(t)\right|^{2} d t-\lambda \int_{\mathbb{R}} F\left(t, u_{n}(t)\right) d t \\
& -\left(a+\left.\left.b \int_{\mathbb{R}}\right|_{-\infty} D_{t}^{\alpha} u_{n}(t)\right|^{2} d t\right)^{\theta-1} \\
& \cdot \int_{\mathbb{R}}\left|-\infty D_{t}^{\alpha} u_{n}(t)\right|^{2} d t-\int_{\mathbb{R}} \mu V(t)\left|u_{n}(t)\right|^{2} d t \\
& +\int_{\mathbb{R}} f\left(t, u_{n}(t)\right) u_{n}(t) d t
\end{aligned}
$$




$$
\begin{aligned}
& =\frac{a \lambda}{2 b \theta}\left(a+\left.\left.b \int_{\mathbb{R}}\right|_{-\infty} D_{t}^{\alpha} u_{n}(t)\right|^{2} d t\right)^{\theta-1} \\
& +\frac{\lambda-2 \theta}{2 \theta}\left(a+\left.\left.b \int_{\mathbb{R}}\right|_{-\infty} D_{t}^{\alpha} u_{n}(t)\right|^{2} d t\right)^{\theta-1} \\
& \left.\left.\cdot \int_{\mathbb{R}}\right|_{-\infty} D_{t}^{\alpha} u_{n}(t)\right|^{2} d t+\frac{\lambda-2}{2} \\
& \cdot \int_{\mathbb{R}} \mu V(t)\left|u_{n}(t)\right|^{2} d t \\
& +\int_{\mathbb{R}}\left(f\left(t, u_{n}(t)\right) u_{n}(t)-\lambda F\left(t, u_{n}(t)\right)\right) d t \\
& \geq\left.\left.\frac{\lambda-2 \theta}{2 \theta} a^{\theta-1} \int_{\mathbb{R}}\right|_{-\infty} D_{t}^{\alpha} u_{n}(t)\right|^{2} d t+\frac{\lambda-2}{2} \\
& \cdot \int_{\mathbb{R}} \mu V(t)\left|u_{n}(t)\right|^{2} d t \geq \frac{\lambda-2 \theta}{2 \theta}\|u\|_{X_{\mu}^{\alpha}}^{2} .
\end{aligned}
$$

Hence, $\left\{u_{n}\right\}_{n \in \mathbb{N}}$ is bounded in $X_{\mu}^{\alpha}$.

So, passing onto subsequence if necessary, thanks to Lemma 3, we have

$$
\begin{aligned}
u_{n} & \rightarrow u, \quad \text { weakly in } X_{\mu}^{\alpha}, \\
u_{n} & \longrightarrow u, \quad \text { strongly a.e. in } \mathbb{R}, \\
u_{n} & \longrightarrow u, \\
\text { strongly a.e. in } L^{s}\left(\mathbb{R}^{N}\right), 2 \leq s<+\infty, & \\
\int_{\mathbb{R}}\left|{ }_{-\infty} D_{t}^{\alpha} u_{n}(t)\right|^{2} d t & \longrightarrow \rho_{1} \geq 0, \\
\int_{\mathbb{R}} \mu V(t)\left|u_{n}\right|^{2} d t & \longrightarrow \rho_{2} \geq 0 .
\end{aligned}
$$

We will prove that

$$
\begin{aligned}
\int_{\mathbb{R}}\left|{ }_{-\infty} D_{t}^{\alpha} u(t)\right|^{2} d t & =\rho_{1}, \\
\int_{\mathbb{R}} \mu V(t)|u|^{2} d x & =\rho_{2} .
\end{aligned}
$$

Let $\varphi \in X_{\mu}^{\alpha}$ be fixed and denote by $B_{\varphi}$ the linear functional on $X_{\mu}^{\alpha}$ defined by

$$
B_{\varphi}(v):=\int_{\mathbb{R}}{ }_{-\infty} D_{t}^{\alpha} \varphi(t) \cdot{ }_{-\infty} D_{t}^{\alpha} v(t) d t,
$$

and set

$$
\Delta_{\alpha}(u):=\left.\left.\int_{\mathbb{R}}\right|_{-\infty} D_{t}^{\alpha} u(t)\right|^{2} d t,
$$

for all $v \in X_{\mu}^{\alpha}$. In view of the Hölder inequality and definition of $B_{\varphi}$, we have

$$
\begin{aligned}
& \left\langle I_{\mu, \theta}^{\prime}\left(u_{n}\right)-I_{\mu, \theta}^{\prime}(u), u_{n}-u\right\rangle=\left(a+b \Delta_{\alpha}\left(u_{n}\right)\right)^{\theta-1} \\
& \text { - } B_{u_{n}}\left(u_{n}-u\right)-\left(a+b \Delta_{\alpha}\left(u_{n}\right)\right)^{\theta-1} B_{u}\left(u_{n}-u\right) \\
& +\int_{\mathbb{R}} \mu V(t)\left(u_{n}-u\right)\left(u_{n}-u\right) d t \\
& -\int_{\mathbb{R}}\left(f\left(t, u_{n}\right)-f(t, u)\right)\left(u_{n}-u\right) d t \geq(a \\
& \left.+b \Delta_{\alpha}\left(u_{n}\right)\right)^{\theta-1} \Delta_{\alpha}\left(u_{n}\right)-\left(a+b \Delta_{\alpha}\left(u_{n}\right)\right)^{\theta-1} \\
& \cdot\left(\Delta_{\alpha}\left(u_{n}\right)\right)^{(\theta-1) / 2}\left(\Delta_{\alpha}\left(u_{n}\right)\right)^{1 / 2}+\left(a+b \Delta_{\alpha}(u)\right)^{\theta-1} \\
& \cdot \Delta_{\alpha}(u)-\left(a+b \Delta_{\alpha}(u)\right)^{\theta-1}\left(\Delta_{\alpha}(u)\right)^{(\theta-1) / 2} \\
& \cdot\left(\Delta_{\alpha}\left(u_{n}\right)\right)^{1 / 2}+\int_{\mathbb{R}} \mu V(t)\left|u_{n}\right|^{2} d t \\
& -\left(\int_{\mathbb{R}} \mu V(t)\left|u_{n}\right|^{2} d t\right)^{1 / 2}\left(\int_{\mathbb{R}} \mu V(t)|u|^{2} d t\right)^{1 / 2} \\
& +\int_{\mathbb{R}} \mu V(t)|u|^{2} d t-\left(\int_{\mathbb{R}} \mu V(t)|u|^{2} d t\right)^{1 / 2} \\
& \cdot\left(\int_{\mathbb{R}} \mu V(t)\left|u_{n}\right|^{2} d t\right)^{1 / 2} \\
& -\int_{\mathbb{R}}\left(f\left(t, u_{n}\right)-f(t, u)\right)\left(u_{n}-u\right) d t=(a \\
& \left.+b \Delta_{\alpha}\left(u_{n}\right)\right)^{\theta-1}\left(\Delta_{\alpha}\left(u_{n}\right)\right)^{(\theta-1) / 2}\left[\left(\Delta_{\alpha}\left(u_{n}\right)\right)^{1 / 2}\right. \\
& \left.-\left(\Delta_{\alpha}(u)\right)^{1 / 2}\right]+\left(a+b \Delta_{\alpha}(u)\right)^{\theta-1}\left(\Delta_{\alpha}(u)\right)^{(\theta-1) / 2} \\
& \cdot\left[\left(\Delta_{\alpha}(u)\right)^{1 / 2}-\left(\Delta_{\alpha}\left(u_{n}\right)\right)^{1 / 2}\right] \\
& +\left(\int_{\mathbb{R}} \mu V(t)\left|u_{n}\right|^{2} d t\right)^{1 / 2}\left[\left(\int_{\mathbb{R}} \mu V(t)\left|u_{n}\right|^{2} d t\right)^{1 / 2}\right. \\
& \left.-\left(\int_{\mathbb{R}} \mu V(t)|u|^{2} d t\right)^{1 / 2}\right]+\left(\int_{\mathbb{R}} \mu V(t)|u|^{2} d t\right)^{1 / 2} \\
& \cdot\left[\left(\int_{\mathbb{R}} \mu V(t)|u|^{2} d t\right)^{1 / 2}\right. \\
& \left.-\left(\int_{\mathbb{R}} \mu V(t)\left|u_{n}\right|^{2} d t\right)^{1 / 2}\right] \\
& -\int_{\mathbb{R}}\left(f\left(t, u_{n}\right)-f(t, u)\right)\left(u_{n}-u\right) d t \\
& =\left[\left(\Delta_{\alpha}\left(u_{n}\right)\right)^{1 / 2}-\left(\Delta_{\alpha}(u)\right)^{1 / 2}\right] \\
& \cdot\left[\left(a+b \Delta_{\alpha}\left(u_{n}\right)\right)^{\theta-1}\left(\Delta_{\alpha}\left(u_{n}\right)\right)^{(\theta-1) / 2}\right. \\
& \left.-\left(a+b \Delta_{\alpha}(u)\right)^{\theta-1}\left(\Delta_{\alpha}(u)\right)^{(\theta-1) / 2}\right]
\end{aligned}
$$




$$
\begin{aligned}
& +\left[\left(\int_{\mathbb{R}} \mu V(t)\left|u_{n}\right|^{2} d t\right)^{1 / 2}\right. \\
& \left.-\left(\int_{\mathbb{R}} \mu V(t)|u|^{2} d t\right)^{1 / 2}\right]^{2} \\
& -\int_{\mathbb{R}}\left(f\left(t, u_{n}\right)-f(t, u)\right)\left(u_{n}-u\right) d t .
\end{aligned}
$$

Since $u_{n} \rightarrow u$ in $X_{\mu}^{\alpha}$ and $I_{\mu, \theta}^{\prime}\left(u_{n}\right) \rightarrow 0$ as $n \rightarrow \infty$ in $\left(X_{\mu}^{\alpha}\right)^{*}$, therefore $\left\langle I_{\mu, \theta}^{\prime}\left(u_{n}\right)-I_{\mu, \theta}^{\prime}(u), u_{n}-u\right\rangle \rightarrow 0$ as $n \rightarrow \infty$. Now, using (F1) and Hölder inequality, we obtain

$$
\begin{aligned}
& \int_{\mathbb{R}}\left(f\left(t, u_{n}\right)-f(t, u)\right)\left(u_{n}-u\right) d t \\
& \leq \int_{\mathbb{R}}\left|c_{0}\left(\left|u_{n}\right|+|u|\right)+\sum_{j=1}^{l} c_{j}\left(\left|u_{n}\right|^{q_{j}-1}+|u|^{q_{j}-1}\right)\right| \\
& \cdot\left|u_{n}-u\right| d x \leq c_{0}\left(\left\|u_{n}\right\|_{L^{2}(\mathbb{R})}+\|u\|_{L^{2}(\mathbb{R})}\right) \| u_{n} \\
& -u \|_{L^{2}(\mathbb{R})}+\sum_{j=1}^{l} c_{j}\left(\left\|u_{n}\right\|_{L^{q_{j}(\mathbb{R})}}^{q_{j}-1}+\left\|u_{n}\right\|_{L^{q_{j}(\mathbb{R})}}^{q_{j}-1}\right) \\
& \cdot\left\|u_{n}-u\right\|_{L^{q_{j}(\mathbb{R})}},
\end{aligned}
$$

which, in view of (39), yields

$$
\lim _{n \rightarrow \infty} \int_{\mathbb{R}}\left(f\left(t, u_{n}\right)-f(t, u)\right)\left(u_{n}-u\right) d t=0
$$

Since $u_{n} \rightarrow u$ a.e. in $\mathbb{R}$, it follows by Fatou's lemma that

$$
\begin{gathered}
\Delta_{\alpha}(u) \leq \liminf _{n \rightarrow \infty} \Delta_{\alpha}\left(u_{n}\right)=\rho_{1} \\
\int_{\mathbb{R}} \mu V(t)|u|^{2} d t \leq \liminf _{n \rightarrow \infty} \int_{\mathbb{R}} \mu V(t)\left|u_{n}\right|^{2} d t=\rho_{2} .
\end{gathered}
$$

Noting that $\Pi(s)=(a+b s)^{\theta-1} s^{(\theta-1) / 2}$ is a nondecreasing function for $s \geq 0$, we get

$$
\begin{aligned}
& {\left[\left(\rho_{1}\right)^{1 / 2}-\left(\Delta_{\alpha}(u)\right)^{1 / 2}\right]\left[\left(a+b \rho_{1}\right)^{\theta-1}\left(\rho_{1}\right)^{(\theta-1) / 2}\right.} \\
& \left.-\left(a+b \Delta_{\alpha}(u)\right)^{\theta-1}\left(\Delta_{\alpha}(u)\right)^{(\theta-1) / 2}\right], \\
& {\left[\left(\rho_{2}\right)^{1 / 2}-\left(\int_{\mathbb{R}} \mu V(t)|u|^{2} d t\right)^{1 / 2}\right]^{2} \geq 0 .}
\end{aligned}
$$

Now, in view of $\left\langle I_{\mu, \theta}^{\prime}\left(u_{n}\right)-I_{\mu, \theta}^{\prime}(u), u_{n}-u\right\rangle \rightarrow 0$ as $n \rightarrow \infty$, (46), and (47), one has

$$
\begin{aligned}
& 0 \geq \liminf _{n \rightarrow \infty}\left\{\left[\left(\Delta_{\alpha}\left(u_{n}\right)\right)^{1 / 2}-\left(\Delta_{\alpha}(u)\right)^{1 / 2}\right]\right. \\
& \cdot\left[\left(a+b \Delta_{\alpha}\left(u_{n}\right)\right)^{\theta-1}\left(\Delta_{\alpha}\left(u_{n}\right)\right)^{(\theta-1) / 2}\right. \\
& \left.-\left(a+b \Delta_{\alpha}(u)\right)^{\theta-1}\left(\Delta_{\alpha}(u)\right)^{(\theta-1) / 2}\right] \\
& +\left[\left(\int_{\mathbb{R}} \mu V(t)\left|u_{n}\right|^{2} d t\right)^{1 / 2}\right. \\
& \left.-\left(\int_{\mathbb{R}} \mu V(t)|u|^{2} d t\right)^{1 / 2}\right]^{2} \\
& \left.-\int_{\mathbb{R}}\left(f\left(t, u_{n}\right)-f(t, u)\right)\left(u_{n}-u\right) d t\right\} \\
& \geq \lim _{n \rightarrow \infty}\left\{\left[\left(\Delta_{\alpha}\left(u_{n}\right)\right)^{1 / 2}-\left(\Delta_{\alpha}(u)\right)^{1 / 2}\right]\right. \\
& \cdot\left[\left(a+b \Delta_{\alpha}\left(u_{n}\right)\right)^{\theta-1}\left(\Delta_{\alpha}\left(u_{n}\right)\right)^{(\theta-1) / 2}\right. \\
& \left.\left.-\left(a+b \Delta_{\alpha}(u)\right)^{\theta-1}\left(\Delta_{\alpha}(u)\right)^{(\theta-1) / 2}\right]\right\} \\
& +\lim _{n \rightarrow \infty}\left[\left(\int_{\mathbb{R}} \mu V(t)\left|u_{n}\right|^{2} d t\right)^{1 / 2}\right. \\
& \left.-\left(\int_{\mathbb{R}} \mu V(t)|u|^{2} d t\right)^{1 / 2}\right]^{2} \\
& -\lim _{n \rightarrow \infty}\left\{\int_{\mathbb{R}}\left(f\left(t, u_{n}\right)-f(t, u)\right)\left(u_{n}-u\right) d t\right\} \\
& \geq\left[\left(\rho_{1}\right)^{1 / 2}-\left(\Delta_{\alpha}(u)\right)^{1 / 2}\right]\left[\left(a+b \rho_{1}\right)^{\theta-1}\left(\rho_{1}\right)^{(\theta-1) / 2}\right. \\
& \left.-\left(a+b \Delta_{\alpha}(u)\right)^{\theta-1}\left(\Delta_{\alpha}(u)\right)^{(\theta-1) / 2}\right]+\left[\left(\rho_{2}\right)^{1 / 2}\right. \\
& \left.-\left(\int_{\mathbb{R}} \mu V(t)|u|^{2} d t\right)^{1 / 2}\right]^{2}
\end{aligned}
$$

Then, from (48)-(49), we get

$$
\begin{aligned}
\Delta_{\alpha}(u) & =\int_{\mathbb{R}}\left|{ }_{-\infty} D_{t}^{\alpha} u(t)\right|^{2} d t=\rho_{1}, \\
\int_{\mathbb{R}} \mu V(t)|u|^{2} d t & =\rho_{2} .
\end{aligned}
$$

Hence, we obtain $\left\|u_{n}\right\|_{X_{\mu}^{\alpha}} \rightarrow\|u\|_{X_{\mu}^{\alpha}}$. As $X_{\mu}^{\alpha}$ is a reflexive Banach space (see Lemma 2), it is isomorphic to a locally uniformly convex space. So the weak convergence and norm convergence imply strong convergence. This completes the proof. 
Let $\left\{e_{j}\right\}$ be a total orthonormal basis of $L^{2}(\mathbb{R})$ and define $X_{j}=\mathbb{R} e_{j}, j \in \mathbb{N}$,

$$
\begin{aligned}
& Y_{k}=\oplus_{j=1}^{k} X_{j}, \\
& Z_{k}=\oplus_{j=k+1}^{\infty} X_{j}, \\
& k \in \mathbb{N} .
\end{aligned}
$$

Lemma 12. Assume that (V1) holds. Then, for $2<p<+\infty$,

$$
\beta_{k}:=\sup _{u \in Z_{k},\|u\|_{X_{\mu}^{\alpha}}=1}\|u\|_{L^{p}(\mathbb{R})} \longrightarrow 0, \quad k \longrightarrow \infty
$$

Proof. The proof is similar to that of Lemma 3.8 in [28]. So it is omitted. that

In view of Lemma 12, we can choose an integer $k \geq 1$ such

$$
\begin{array}{r}
\int_{\mathbb{R}}|u|^{2} d t \\
\leq \frac{1}{2 c_{0}}\left(\int_{\mathbb{R}} a\left(\left.\left.\right|_{-\infty} D_{t}^{\alpha} u(\mathrm{t})\right|^{2}\right) d t+\int_{\mathbb{R}} \mu V(t)|u|^{2} d t\right) \\
\forall u \in Z_{m} \cap X_{\mu}^{\alpha},
\end{array}
$$

where $c_{1}$ is a constant given in condition (F1). Let

$$
\mathfrak{R}(t)= \begin{cases}1, & |t|>r, \\ 0, & |t| \leq r,\end{cases}
$$

and set $Y=\left\{(1-\Re) u: u \in X_{\mu}^{\alpha}\right.$, $\left.(1-\Re) u \in Y_{k}\right\}$ and $Z=$ $\left\{(1-\mathfrak{R}) u: u \in X_{\mu}^{\alpha}\right.$, $\left.(1-\mathfrak{R}) u \in Z_{k}\right\}+\left\{\Re v: v \in X_{\mu}^{\alpha}\right\}$. Hence $Y$ and $Z$ are subspaces of $X_{\mu}^{\alpha}$, and $X_{\mu}^{\alpha}=Y \oplus Z$.

Lemma 13. Suppose that (V1), (V2), and (F1) are satisfied. Then there exist constants $\varrho, \beta>0$ such that $\left.I_{\mu, \theta}\right|_{\partial B_{e} \cap Z} \geq \alpha$.

Proof. In view of (V2), (53), and definition of the space $Z$, we have

$$
\begin{aligned}
\|u\|_{L^{2}(\mathbb{R})}^{2}= & \int_{|t|<r}|u(t)|^{2} d t+\int_{|t| \geq r}|u(t)|^{2} d t \\
\leq & \frac{1}{2 c_{0}}\|u\|_{X_{\mu}^{\alpha}}^{2} \\
& +\frac{1}{\mu \omega} \int_{\{t \in \mathbb{R}, V(t)>\omega\}} \mu V(t)|u(t)|^{2} d t \\
\leq & \frac{1}{2 c_{0}}\|u\|_{X_{\mu}^{\alpha}}^{2}+\frac{1}{\mu \omega}\|u\|_{X_{\mu}^{\alpha}}^{2} \quad \forall u \in Z .
\end{aligned}
$$

Therefore, from (23), (55), and (F1) and for large enough value of $\mu$, we get

$$
\begin{aligned}
I_{\mu, \theta}(u)= & \frac{1}{2 b \theta}\left(a+b \Delta_{\alpha}(u)\right)^{\theta}+\frac{1}{2} \int_{\mathbb{R}} \mu V(t)|u|^{2} d t \\
& -\int_{\mathbb{R}} F(t, u) d t \\
\geq & \frac{a^{\theta-1}}{2} \Delta_{\alpha}(u)+\frac{1}{2} \int_{\mathbb{R}} \mu V(t)|u|^{2} d t \\
& -\int_{\mathbb{R}} F(t, u) d t \\
\geq & \frac{1}{2}\|u\|_{X_{\mu}^{\alpha}}^{2}-\frac{c_{0}}{2}\|u\|_{L^{2}(\mathbb{R})}^{2}-\sum_{j=1}^{l} \frac{c_{j}}{q_{j}}\|u\|_{L^{q_{j}}(\mathbb{R})}^{q_{j}} \\
\geq & \frac{1}{4}\|u\|_{X_{\mu}^{\alpha}}^{2}-\frac{c_{0}}{\mu \omega 2}\|u\|_{X_{\mu}^{\alpha}}^{2}-\sum_{j=1}^{l} \frac{c_{j} D_{q_{j}}^{q_{j}}}{q_{j}}\|u\|_{X_{\mu}^{\alpha}}^{q_{j}} \\
\geq & \frac{1}{8}\|u\|_{X_{\mu}^{\alpha}}^{2}-\sum_{j=1}^{l} \frac{c_{j} D_{q_{j}}^{q_{j}}}{q_{j}}\|u\|_{X_{\mu}^{\alpha}}^{q_{j}} .
\end{aligned}
$$

Since $2<q_{j}(j=1, \ldots, l)$, there exist constants $\varrho, \beta>0$ such that $\left.I_{\mu, \theta}\right|_{\partial B_{e} \cap Z} \geq \beta$.

Lemma 14. Assume that (F1) and (F4) are satisfied. Then, for any finite dimensional subspace $\widetilde{X}_{\mu}^{\alpha} \subset X_{\mu}^{\alpha}$, there is $R=$ $R\left(\widetilde{X}_{\mu}^{\alpha}\right)>0$ such that $I_{\mu, \theta}(u) \leq 0$ on $\widetilde{X}_{\mu}^{\alpha} \backslash B_{R}$.

Proof. Since all the norms in the finite dimensional space are equivalent, there exists a constant $\Upsilon$ such that

$$
\|u\|_{L^{2 \theta(\mathbb{R})}} \geq \Upsilon\|u\|_{X_{\mu}^{\alpha}}, \quad \forall u \in \widetilde{X}_{\mu}^{\alpha} .
$$

From (F1) and (F4), for any $L>b^{\theta-1} / 2 \theta \Upsilon^{2 \theta} a^{\theta(\theta-1)}$, there exists a constant $C_{L}>0$ such that

$$
F(t, u) \geq L|u|^{2 \theta}-C_{L}|u|^{2}, \quad \forall(t, u) \in \mathbb{R} \times \mathbb{R} .
$$

Thus

$$
\begin{aligned}
I_{\mu, \theta}(u)= & \frac{1}{2 b \theta}\left(a+b \Delta_{\alpha}(u)\right)^{\theta}+\frac{1}{2} \int_{\mathbb{R}} \mu V(t)|u|^{2} d t \\
& -\int_{\mathbb{R}} F(t, u) d t \\
\leq & \frac{1}{2 b \theta}\left(a+\frac{b}{a^{\theta-1}}\|u\|_{X_{\mu}^{\alpha}}^{2}\right)^{\theta}+\frac{1}{2}\|u\|_{X_{\mu}^{\alpha}}^{2} \\
& +C_{L}\|u\|_{L^{2}(\mathbb{R})}^{2}-L\|u\|_{L^{2 \theta}(\mathbb{R})}^{2 \theta} \\
\leq & \frac{1}{2 b \theta}\left(a+\frac{b}{a^{\theta-1}}\|u\|_{X_{\mu}^{\alpha}}^{2}\right)^{\theta} \\
& +\left(\frac{1}{2}+C_{L} D_{2}^{2}\right)\|u\|_{X_{\mu}^{\alpha}}^{2}-L Y^{2 \theta}\|u\|_{X_{\mu}^{\alpha}}^{2 \theta}
\end{aligned}
$$

for all $u \in \widetilde{X}_{\mu}^{\alpha}$. Consequently, there is a large $R>0$ such that $I_{\mu, \theta}(u) \leq 0$ on $\widetilde{X}_{\mu}^{\alpha} \backslash B_{R}$. Therefore, the proof is completed. 


\section{Existence of Weak Solutions}

In this section, we present our main results.

Theorem 15. Assume that (V1), (V2), (F1), (F3), (F4), and (F5) hold. Then problem (1) has infinitely many nontrivial weak solutions whenever $\mu>0$ is sufficiently large.

Proof. We know that $I_{\mu, \theta}(0)=0$, and it is even by (F5). Let $X=X_{\mu}^{\alpha}$ and $Y$ and $Z$ be as defined in Section 2. By Lemmas 11,13 , and 14 , it follows that $I_{\mu, \theta}$ satisfies all the condition of the Theorem 10. Therefore, problem (1) has infinitely many nontrivial weak solutions whenever $\mu>0$ is sufficiently large.

Theorem 16. Assume that (V1), (V2), (F1), (F2), (F3), and (F4) hold. Then problem (1) has at least one nontrivial weak solution when $\mu>0$.

Proof. We complete the proof in three steps.

Step 1. Clearly $I_{\mu, \theta}(0)=0$ and $I_{\mu, \theta} \in C^{1}\left(X_{\mu}^{\alpha}, \mathbb{R}\right)$ satisfies the (PS) condition by Lemma 11.

Step 2. It will be shown that there exist constants $\varrho, \beta>0$ such that $I_{\mu, \theta}$ satisfies condition (i) of Theorem 9. For any $\varepsilon>0$, by (F1) and (F2), there exists a constant $c_{\varepsilon}>0$ such that

$$
|F(t, u)| \leq \frac{\epsilon}{2}|u|^{2}+\sum_{j=1}^{l} \frac{c_{j}^{\varepsilon}}{q_{j}}|u|^{q_{j}} .
$$

Thus, by (23) and (60), for small $\rho>0$, we get

$$
\begin{aligned}
I_{\mu, \theta}(u)= & \frac{1}{2 b \theta}\left(a+b \Delta_{\alpha}(u)\right)^{\theta}+\frac{1}{2} \int_{\mathbb{R}} \mu V(t)|u|^{2} d t \\
& -\int_{\mathbb{R}} F(t, u) d t \\
\geq & \frac{a^{\theta-1}}{2} \Delta_{\alpha}(u)+\frac{1}{2} \int_{\mathbb{R}} \mu V(t)|u|^{2} d t \\
& -\int_{\mathbb{R}} F(t, u) d t \\
\geq & \frac{1}{2}\left(\|u\|_{X_{\mu}^{\alpha}}^{2}-\varepsilon D_{2}^{2}\|u\|_{X_{\mu}^{\alpha}}^{2}\right)-\sum_{j=1}^{l} \frac{c_{j}^{\varepsilon}}{q_{j}} D_{q_{j}}^{q_{j}}\|u\|_{X_{\mu}^{\alpha}}^{q_{j}} \\
\geq & \frac{1}{8}\left(1-\varepsilon D_{2}^{2}\right) \varrho^{2},
\end{aligned}
$$

for all $u \in \bar{B}_{\varrho}$, where $B_{\varrho}=\left\{u \in X_{\mu}^{\alpha}:\|u\|_{X_{\mu}^{\alpha}}<\varrho\right\}$. So it suffices to choose $\varepsilon=1 / 2 D_{2}^{2}$ so that

$$
\left.I_{\mu, \theta}\right|_{\partial B_{e}} \geq \frac{1}{16} \varrho^{2}:=\beta>0 .
$$

Step 3. It remains to prove that there exists an $e \in X_{\mu}^{\alpha}$ such that $\|u\|_{X_{\mu}^{\alpha}}>\varrho$ and $I_{\mu, \theta}(e) \leq 0$, where $\rho$ is defined in Step 2. Let us consider

$$
\begin{aligned}
I_{\mu, \theta}(\sigma u)= & \frac{1}{2 b \theta}\left(a+b \sigma^{2} \Delta_{\alpha}(u)\right)^{\theta} \\
& +\frac{\sigma^{2}}{2} \int_{\mathbb{R}} \mu V(t)|u|^{2} d t-\int_{\mathbb{R}} F(t, \sigma u) d t,
\end{aligned}
$$

for all $\sigma \in \mathbb{R}$. Take $0 \neq u \in X_{\mu}^{\alpha}$. By (F1) and (F4), for any $\kappa>b^{\theta-1}\left(\Delta_{\alpha}(u)\right)^{\theta} / 2 \theta \int_{\mathbb{R}}|u|^{2 \theta} d t$, there is a constant $C_{\kappa}>0$ such that

$$
F(t, u) \geq \kappa|u|^{2 \theta}-C_{\kappa}|u|^{2} .
$$

So we have

$$
\begin{aligned}
I_{\mu, \theta}(\sigma u) \leq & \frac{1}{2 b \theta}\left(a+b \sigma^{2} \Delta_{\alpha}(u)\right)^{\theta} \\
& +\frac{\sigma^{2}}{2} \int_{\mathbb{R}} \mu V(t)|u|^{2} d t+C_{\kappa} \sigma^{2} \int_{\mathbb{R}}|u|^{2} d t \\
& -\kappa \sigma^{2 \theta} \int_{\mathbb{R}}|u|^{2 \theta} d t \longrightarrow-\infty,
\end{aligned}
$$

as $\sigma \rightarrow+\infty$. Thus, there is a point $e \in X_{\mu}^{\alpha} \backslash \bar{B} \varrho$ such that $I_{\mu, \theta}(e) \leq 0$. By Theorem $9, I_{\mu, \theta}$ possesses a critical value $c \geq$ $\alpha>0$ given by

$$
\left.c=\inf _{\gamma \in \Gamma \in[0,1]} \max _{s, \theta} I_{\mu, \theta}(\gamma)\right),
$$

where

$$
\Gamma=\{\gamma \in C([0,1], X): \gamma(0)=0, \gamma(1)=e\} .
$$

Hence there is $u \in X_{\mu}^{\alpha}$ such that $I_{\mu, \theta}(u)=c$ and $I_{\mu, \theta}^{\prime}(u)=0$; that is, problem (1) has a nontrivial weak solution in $X_{\mu}^{\alpha}$.

Theorem 17. Assume that (V1), (V3), (F5), (F6), and (F7) hold. Then problem (1) has infinitely many nontrivial weak solutions for $\mu>0$.

Proof. One can obtain the proof by employing the method of proof for Theorem 15 and using Lemma 7.

\section{Conflicts of Interest}

The authors declare that there are no conflicts of interest regarding the publication of this paper.

\section{Acknowledgments}

This project was supported by National Natural Science Foundation of China (11671339).

\section{References}

[1] S. Ş. Bayın, "Time fractional Schrödinger equation: fox's Hfunctions and the effective potential," Journal of Mathematical Physics, vol. 54, Article ID 012103, 2013. 
[2] J. L. Dubbeldam, Z. Tomovski, and T. Sandev, "Space-time fractional Schrödinger equation with composite time fractional derivative," Fractional Calculus and Applied Analysis, vol. 18, no. 5, pp. 1179-1200, 2015.

[3] A. Iomin, "Fractional-time Schrödinger equation: fractional dynamics on a comb," Chaos, Solitons \& Fractals, vol. 44, no. 4-5, pp. 348-352, 2011.

[4] N. Laskin, "Fractional Schrödinger equation," Physical Review E. Statistical, Nonlinear, and Soft Matter Physics, vol. 66, Article ID 056108, 2002.

[5] M. Naber, “Time fractional Schrödinger equation," Journal of Mathematical Physics, vol. 45, no. 8, pp. 3339-3352, 2004.

[6] N. Nyamoradi and Y. Zhou, "Existence of solutions for a Kirchhoff type fractional differential equations via minimal principle and Morse theory," Topological Methods in Nonlinear Analysis, vol. 46, no. 2, pp. 617-630, 2015.

[7] M. Xiang and B. Zhang, "Degenerate Kirchhoff problems involving the fractional $p$-Laplacian without the (AR) condition," Complex Variables and Elliptic Equations, vol. 60, no. 9, pp. 1277-1287, 2015.

[8] M. Xiang, B. Zhang, and M. Ferrara, "Existence of solutions for Kirchhoff type problem involving the non-local fractional $p$ Laplacian," Journal of Mathematical Analysis and Applications, vol. 424, no. 2, pp. 1021-1041, 2015.

[9] Y. Zhou and L. Peng, "On the time-fractional Navier-Stokes equations," Computers \& Mathematics with Applications, vol. 73, no. 6, pp. 874-891, 2017.

[10] Y. Zhou and L. Peng, "Weak solutions of the time-fractional Navier-Stokes equations and optimal control," Computers \& Mathematics with Applications, vol. 73, no. 6, pp. 1016-1027, 2017.

[11] J. Wang, M. Fečkan, and Y. Zhou, "A survey on impulsive fractional differential equations," Fractional Calculus and Applied Analysis, vol. 19, no. 4, pp. 806-831, 2016.

[12] J. Wang, M. Fĕckan, and Y. Zhou, "Center stable manifold for planar fractional damped equations," Applied Mathematics and Computation, vol. 296, pp. 257-269, 2017.

[13] Y. Zhou, B. Ahmad, and A. Alsaedi, "Existence of nonoscillatory solutions for fractional neutral differential equations," Applied Mathematics Letters, vol. 72, pp. 70-74, 2017.

[14] Y. Zhou, V. Vijayakumar, and R. Murugesu, "Controllability for fractional evolution inclusions without compactness," Evolution Equations and Control Theory, vol. 4, no. 4, pp. 507-524, 2015.

[15] G. A. Mendez Cruz and C. E. Torres Ledesma, "Multiplicity of solutions for fractional Hamiltonian systems with LiouvilleWeyl fractional derivatives," Fractional Calculus and Applied Analysis, vol. 18, no. 4, pp. 875-890, 2015.

[16] C. Torres, "Existence of solutions for fractional Hamiltonian systems," Electronic Journal of Differential Equations, vol. 2013, article 259, pp. 1-12, 2013.

[17] Y. Zhou and L. Zhang, "Existence and multiplicity results of homoclinic solutions for fractional Hamiltonian systems," Computers \& Mathematics with Applications, vol. 73, no. 6, pp. 1325-1345, 2017.

[18] A. Iomin, "Fractional-time quantum dynamics," Physical Review E - Statistical, Nonlinear, and Soft Matter Physics, vol. 80, no. 2, Article ID 022103, 2009.

[19] N. Laskin, "Fractional quantum mechanics," Physical Review E - Statistical, Nonlinear, and Soft Matter Physics, vol. 62, no. 3, pp. 3135-3145, 2000.

[20] F. Cammaroto and L. Vilasi, "On a Schrödinger-Kirchhoff-type equation involving the $p(x)$-Laplacian," Nonlinear Analysis. Theory, Methods \& Applications, vol. 81, pp. 42-53, 2013.
[21] Y. Guo and J. Nie, "Existence and multiplicity of nontrivial solutions for $p$-Laplacian Schrödinger-Kirchhoff-type equations," Journal of Mathematical Analysis and Applications, vol. 428, no. 2, pp. 1054-1069, 2015.

[22] J. Nie, "Existence and multiplicity of nontrivial solutions for a class of Schrödinger-Kirchhoff-type equations," Journal of Mathematical Analysis and Applications, vol. 417, no. 1, pp. 6579, 2014.

[23] P. Pucci, M. Xiang, and B. Zhang, "Multiple solutions for nonhomogeneous Schrödinger-Kirchhoff type equations involving the fractional $p$-Laplacian in $\mathbb{R}^{N}$," Calculus of Variations and Partial Differential Equations, vol. 54, no. 3, pp. 2785-2806, 2015.

[24] A. A. Kilbas, H. M. Srivastava, and J. J. Trujillo, Theory and Applications of Fractional Differential Equations, Elsevier Science B.V., Amsterdam, the Netherlands, 2006.

[25] C. A. Stuart, Bifurcation into Spectral Gaps, Société Mathématique de Belgique, 1995.

[26] P. H. Rabinowitz, Minimax Methods in Critical Point Theory with Applications to Differential Equations, vol. 65, American Mathematical Society, Providence, RI, USA, 1986.

[27] F. Jiao and Y. Zhou, "Existence of solutions for a class of fractional boundary value problems via critical point theory," Computers \& Mathematics with Applications, vol. 62, no. 3, pp. 1181-1199, 2011.

[28] M. Willem, Minimax Theorems, Birkhäuser, Boston, Mass, USA, 1996. 


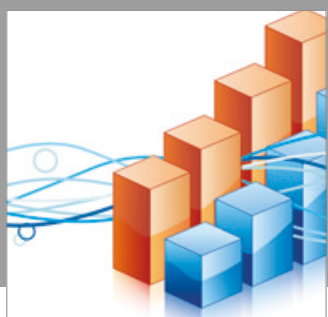

Advances in

Operations Research

vatersals

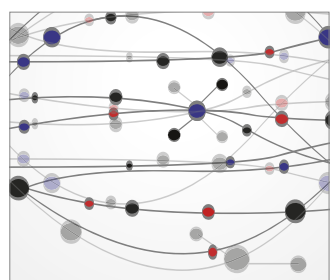

\section{The Scientific} World Journal
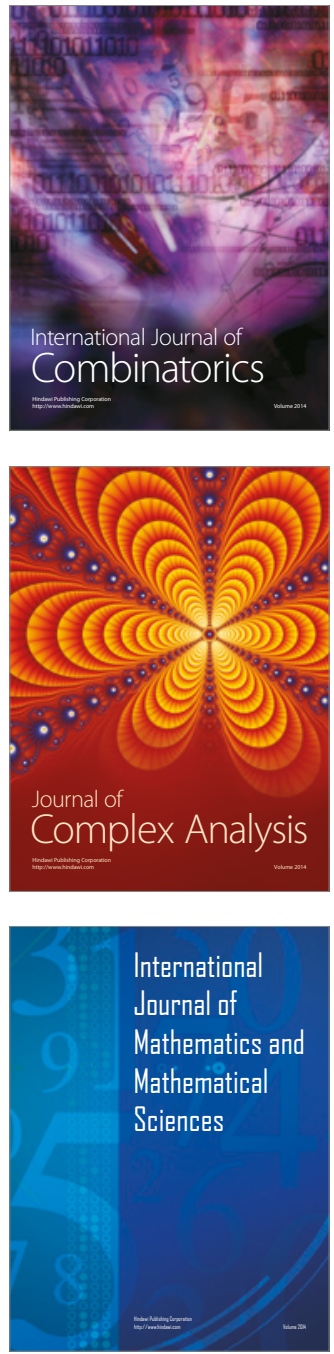
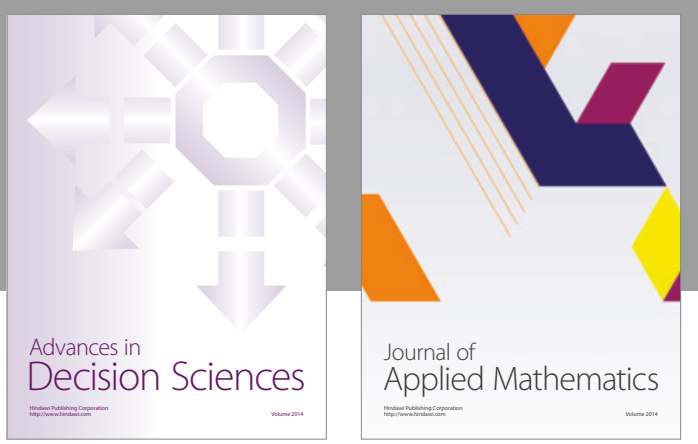

Algebra

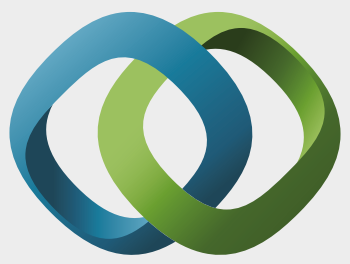

\section{Hindawi}

Submit your manuscripts at

https://www.hindawi.com
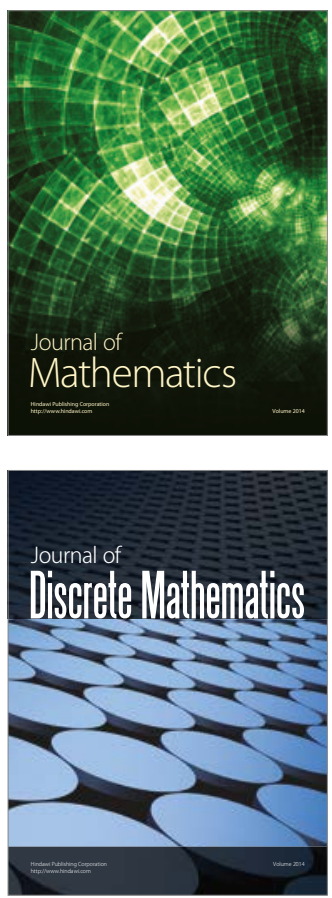

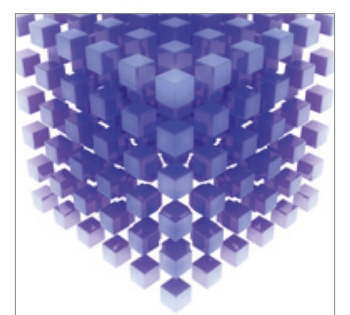

Mathematical Problems in Engineering
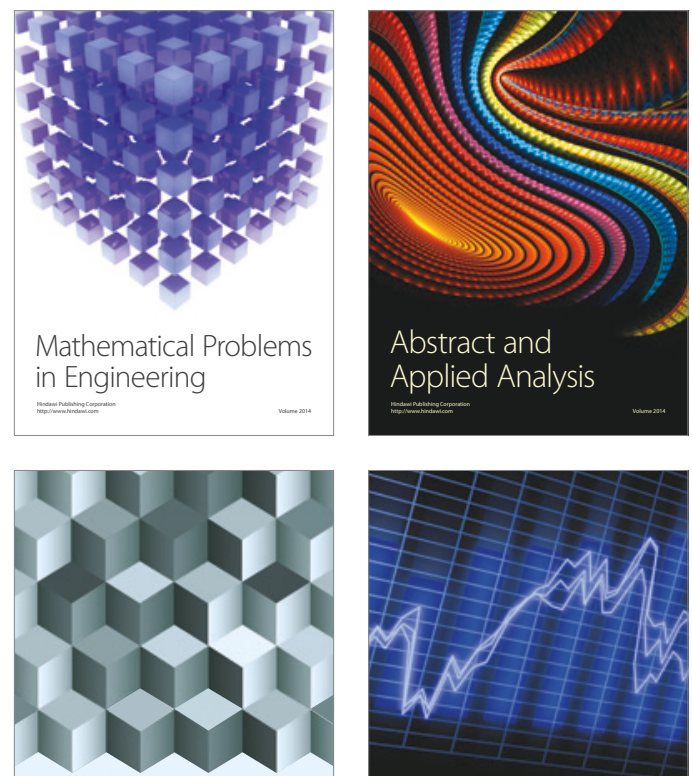

Journal of

Function Spaces

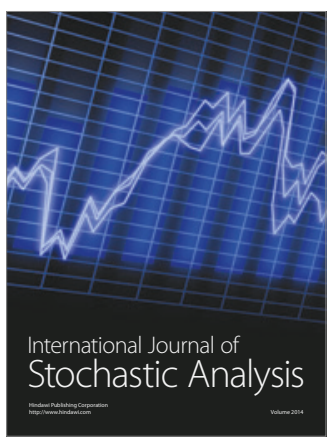

Probability and Statistics
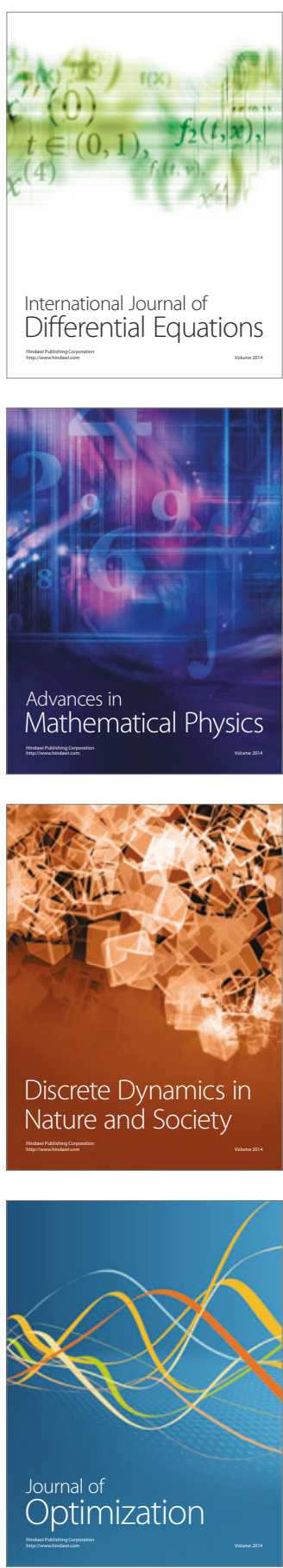